\title{
Long-Term Effect on HbA1c in Poorly Controlled Diabetic Patients Following Nonmydriatic Retinal Image Review at the Time of Endocrinology Visit
}

Lloyd Paul Aiello, MD, PhD, ${ }^{1,2}$ Jerry Cavallerano, OD, PhD, ${ }^{1}$ Jennifer Sun, MD, MPH, ${ }^{1,2}$ Nisreen Salti, $P h D_{1}^{3}$

Mona Nasrallah, $M D^{4}$ Carl Joe Mehanna, $M D_{,}^{5}$

Nasrine Anais El Salloukh, $M D^{5}$ and Haytham I. Salti, $M D^{5}$

${ }^{1}$ Joslin Diabetes Center, Beetham Eye Institute, Boston,

Massachusetts, USA.

${ }^{2}$ Department of Ophthalmology, Harvard Medical School, Boston,

Massachusetts, USA.

${ }^{3}$ Department of Economics, Faculty of Arts and Sciences,

American University of Beirut, Beirut, Lebanon.

${ }^{4}$ Divison of Endocrinology and Metabolism, Department

of Internal Medicine, American University of Beirut

Medical Center, Beirut, Lebanon.

${ }^{5}$ Department of Ophthalmology, American University of Beirut Medical Center, Beirut, Lebanon.

\section{Abstract}

Background: Patient education demonstrates variable benefits on diabetes control.

Introduction: To examine the effect of discussing nonmydriatic retinal imaging findings during a single endocrinology visit on $\mathrm{HbA1c}$ levels after 6, 12, and 60 months.

Materials and Methods: Patients with $\mathrm{HbA1C}>8.0 \%$ and diabetic retinopathy were previously recruited for a prospective study looking at the change in HbA1c at 3 months between those assigned to a session of nonmydriatic imaging with discussion of retinal findings and those assigned to routine endocrinology evaluation alone. The patients were subsequently evaluated at 6, 12, and 60 months after the initial intervention.

Results: Fifty-three of the 57 originally recruited intervention subjects (93\%) and 48 of 54 subjects in the original control group (89\%) were evaluated at 6 and 12 months and 44 patients in each group (75\% and 81\%, respectively) at 60 months. At 6 months, the intervention group maintained larger decreases in median HbA1c compared to control (-1.1 vs. -0.3 , respectively, $\mathrm{p}=0.002$ ) with a trend persisting at 12 months (-0.6 vs. -0.2 , respectively, $\mathrm{p}=0.07)$. After 60 months, there was no significant difference in the median change in HbA1c between treatment and control groups 10.3 vs. 0.1 , respectively, $\mathrm{p}=0.54$ ).
Discussion: The short-term improvement in $\mathrm{HbA1c}$ resulting from discussion of retinal findings persists throughout the first year in this diabetic cohort, but its magnitude declines with time and becomes statistically insignificant at some point between 6 and 12 months.

Conclusions: In patients with poorly controlled diabetes, retinal imaging review may help improve glycemic control but may require repetition periodically for benefit beyond 6 months.

Keywords: diabetic retinopathy, nonmydriatic retinal imaging, glycemic control, telemedicine

\section{Introduction}

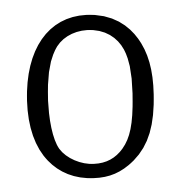

ptimizing blood glucose control is fundamental to reducing complications associated with diabetes. Educational efforts have demonstrated variable benefit on subsequent glycemic control as measured by HbA1c. ${ }^{1,2}$ We previously demonstrated a positive effect on short-term (3-months) glycemic control using a noninvasive imaging intervention that educates patients about their retinopathy findings during their endocrinology visit. ${ }^{3}$ In this study, we report the longer-term follow-up of glycemic control at 6,12, and 60 months following this single original education session.

\section{Materials and Methods}

The study protocol was approved by the Institutional Review Board of the American University of Beirut Medical Center (AUBMC) and was in accordance with the Helsinki declaration. Written informed consent was obtained for all subjects. This study was registered at the ANCTR website with trial registration number ANCTR126 09000362291 . This trial invited the original 111 subjects from the initial prospective study and measured HbA1c level at the AUBMC laboratory 6, 12, and 60 months after the initial intervention.

The "intervention" has been described previously. ${ }^{3}$ Briefly, among a cohort of 111 subjects with poorly controlled diabetes and documented retinopathy, a randomized group of 57 
received nonmydriatic retinal imaging performed by a single technician during their endocrinologist evaluation, followed by viewing of their own images and a brief discussion of the findings by a single retina specialist (H.S.). The retina specialist showed the subjects their images on a computer monitor highlighting clinical findings. Discussion explicitly included the following: (1) presentation demonstrating a range of diabetic retinopathy (DR), including a normal fundus, mild-moderate nonproliferative DR, active proliferative DR, and tractional retinal detachment (Fig. 1); (2) a brief summary of the results of the Diabetes Control and Complications Trial ${ }^{4}$ and the United Kingdom Prospective Diabetes Study ${ }^{5}$ regarding $\mathrm{HbA1c}$ control and DR; and (3) emphasis on the importance of blood glucose control to delay onset or slow progression of DR. The rest of the patients had their standard conventional endocrinology evaluation without the added personalized session.

As previously reported, 3 months after the endocrinologist visit, $\mathrm{HbA1c}$ measurement was performed for each subject and shown to be significantly lower in the intervention group. ${ }^{3}$ In the current study, we report the HbA1c data in these subjects 6,12 , and 60 months after the single original intervention. All blood tests were performed at the same laboratory using the same technique throughout the duration of the study (VARIANT $^{\mathrm{TM}}$ II and D-10 Hemoglobin Testing Systems by Bio-Rad using high performance liquid chromatography).

\section{STATISTICAL ANALYSIS}

Statistical analysis was performed using SAS software v.9.4 (SAS Institute, Inc., Cary, NC). Changes in HbA1c were calculated after 6, 12, and 60 months and compared across treatment and control groups using a Wilcoxon signed-rank test. The baseline HbA1c level was the preintervention measured HbA1c. We also use logistic regression to model the relationship between the intervention and the change in HbA1c while controlling for baseline HbA1c. $p<0.05$ was considered statistically significant.

\section{Results}

Of the 57 original intervention subjects, 53 (93\%) received repeat $\mathrm{HbA} 1 \mathrm{c}$ evaluation at 6 and 12 months and $44(77 \%)$ at 60 months. From the original control group of 54 patients, 48 (89\%) received repeat $\mathrm{HbA} 1 \mathrm{c}$ at 6 and 12 months and 44 (81\%) at 60 months (Fig. 2). Table 1 shows baseline characteristics of
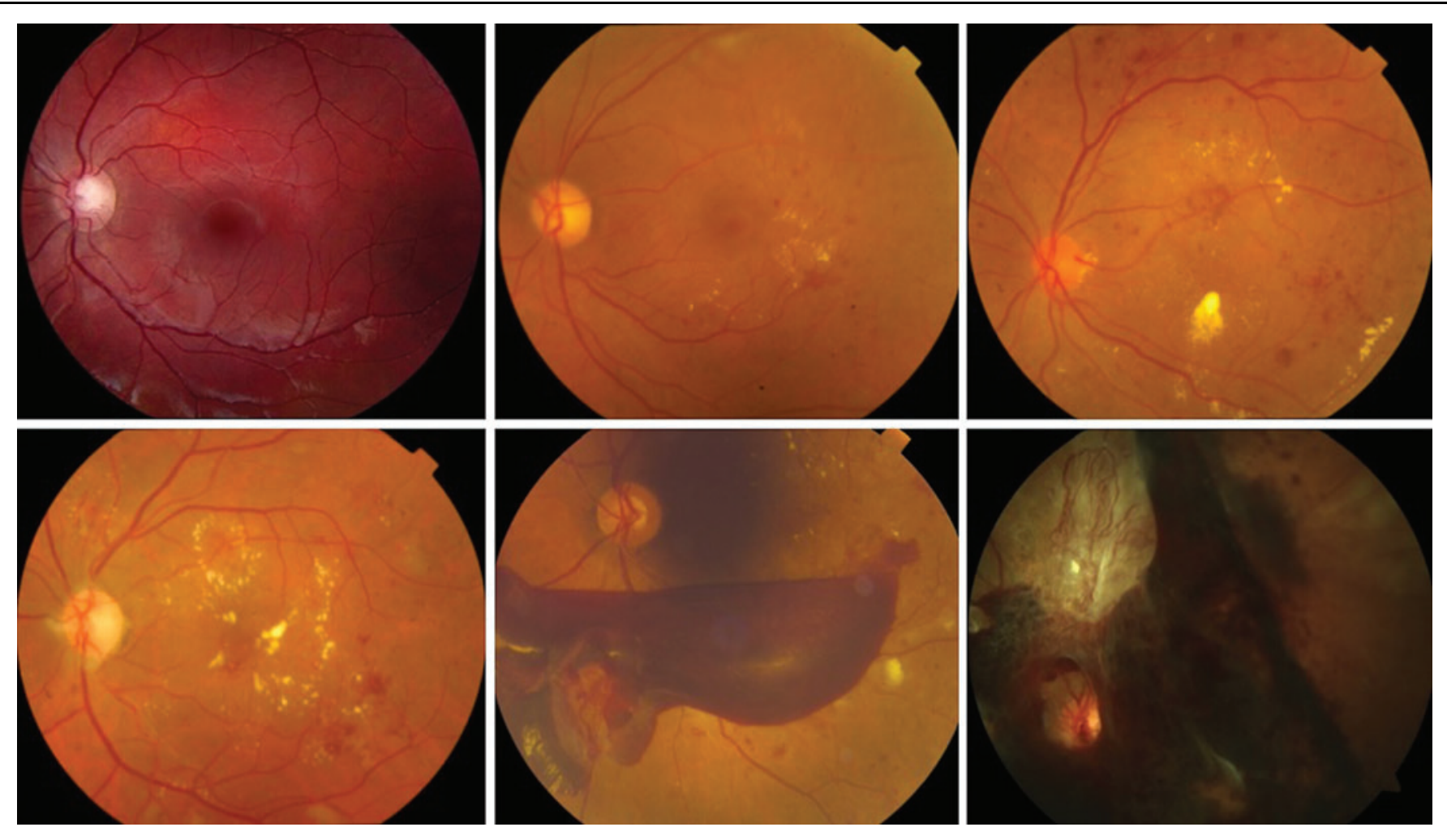

Fig. 1. Images presented at the time of the intervention demonstrating the full spectrum of diabetic retinopathy. Color images are available online. 


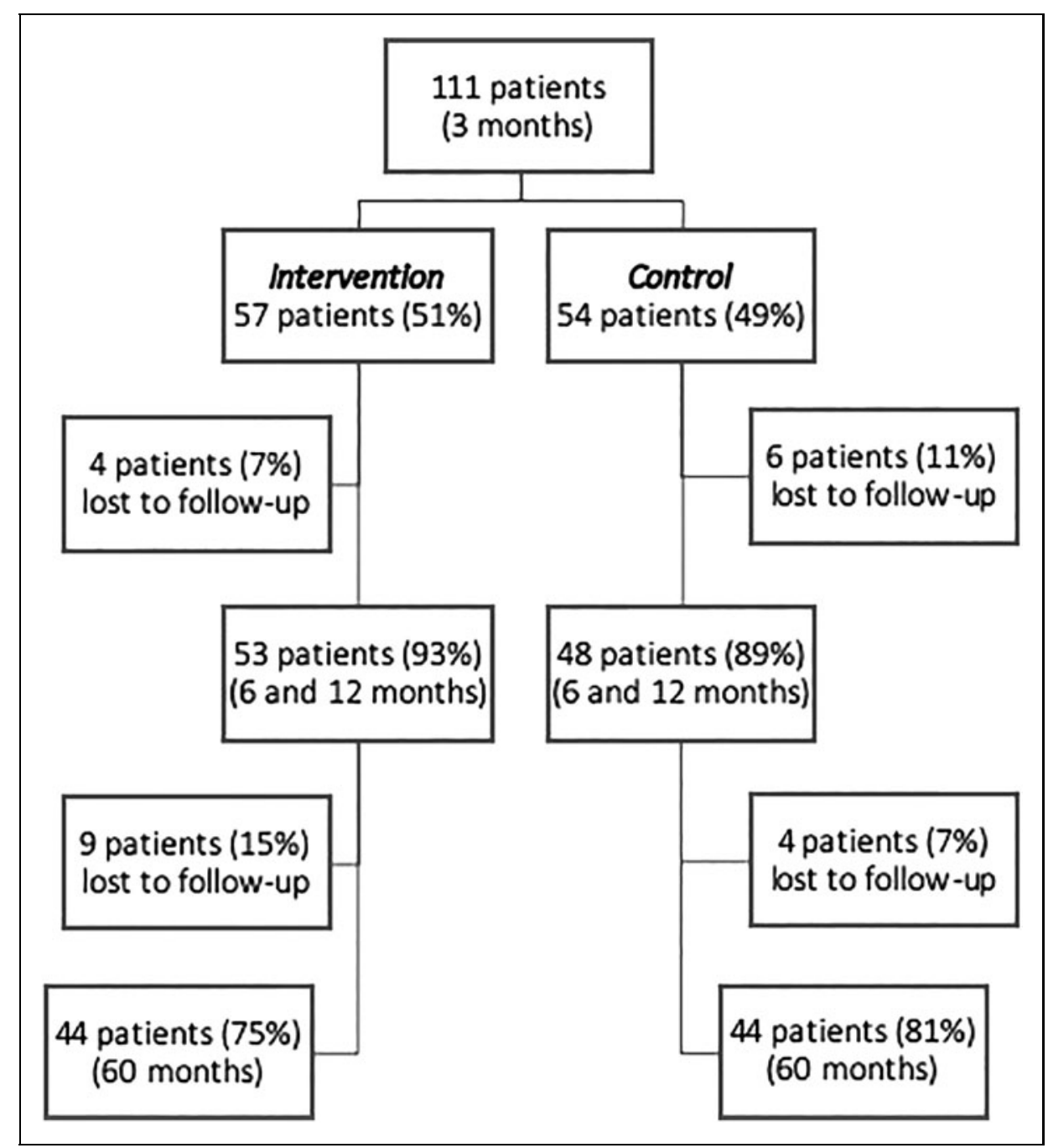

Fig. 2. Follow-up flow diagram.

the follow-up cohort. There were no significant differences between the intervention and control subjects at any followup period. Furthermore, there were no substantial differences in clinical or sociodemographic characteristics between the follow-up and original cohorts. ${ }^{3}$

$\mathrm{HbA1c}$ was reduced to a greater extent in the intervention group than in the control group (Fig. 3) at 3 months (median change -1.4 vs. $+0.2, p=0.001)$, 6 months $(-1.1$ vs. -0.3 , $p=0.002)$, and 12 months ( -0.6 vs. $-0.2, p=0.07)$. The magnitude of benefit was greatest at 3 months following intervention and decreased steadily at the 6- and 12-month follow-up visits. After 60 months, the HbA1c was no longer improved from baseline (median change 0.3 vs. 0.1 ) and was also not statistically different between intervention and control ( $p=0.54)$. The benefit of the intervention on HbA1c reduction remained statistically significant at 6 months even when controlling baseline $\mathrm{HbA} 1 \mathrm{c}$.

\section{Discussion}

Vision loss from DR remains a major cause of visual impairment in the working-age population in most developed countries. It is well established that glycemic control can markedly reduce the development and progression of DR. ${ }^{3,5}$ In addition, studies have demonstrated that vision loss is one of the most feared of all complications from diabetes. ${ }^{6,7}$ We demonstrated previously the significant effect of a single intervention at the time of endocrinology evaluation using a subject's own retinal fundus images and brief retinopathy education for improving diabetes control as measured by subsequent $\mathrm{HbA} 1 \mathrm{c}$ at 3 months. ${ }^{1}$ In this study, we were able to reexamine $\mathrm{HbA} 1 \mathrm{c}$ in $\sim 93 \%$ of the original cohort at 6 and 12 months and $\sim 80 \%$ of the cohort after 60 months. Interestingly, despite no additional intervention, these data demonstrate a continued $\mathrm{HbA} 1 \mathrm{c}$ benefit at 6 months and a trend suggesting persistence at 12 months after the original intervention session.

The magnitude of HbA1c reduction diminished consistently with longer duration of follow-up. After 60 months of follow-up, the intervention effect was no longer statistically significant and the mean $\mathrm{HbA1c}$ returned to similar levels than at study entry. These data suggest that further reinforcement with subsequent interventions may be needed at yearly intervals, although it remains unknown how effective subsequent interventions would be compared to the original effort.

The fact that a single intervention could have such longlasting effects is surprising. Several aspects of the interventional approach and the particular subject cohort may account for this. It is well established that vision loss ranks among the greatest fears in patient populations, sometimes feared even more than death. ${ }^{6,7}$ The intervention in this study directly presented the range of retinal pathology that can occur from DR and then compared that to the current 


\section{Table 1. Baseline characteristics}

\section{AT 6 AND 12 MONTHS}

INTERVENTION CONTROL INTERVENTION CONTROL

GROUP $(n=53)$ GROUP $(n=48)$ GROUP $(n=44)$ GROUP $(n=44)$

\begin{tabular}{l|c|c|c|c}
\hline Age (years), mean \pm SD & 54.5 & 55.5 & 53.8 & 54.6 \\
\hline Female (\%) & 59.6 & 64.5 & 58.1 & 65.9 \\
\hline $\begin{array}{l}\text { Diabetes duration } \\
\text { (years), mean } \pm \text { SD }\end{array}$ & 9.3 & 9.9 & 9.0 & 9.8 \\
\hline Hypertension (\%) & 55.8 & 60.4 & 58.1 & 56.8 \\
\hline Insulin use (\%) & 36.5 & 43.8 & 37.2 & 43.2 \\
\hline Initial HbA1c, mean \pm SD & 10.2 & 9.9 & 10.1 & 10.0 \\
\hline
\end{tabular}

All $p$-values $>0.05$.

$\mathrm{SD}$, standard deviation.

image of the subject themselves. Ways to minimize potential vision loss were then discussed, including the optimization of glycemic control.

Nonmydriatic digital imaging technology allows retinal image acquisition at the time of a patient's endocrinologist visit to be feasible, repeatable, and accurate. ${ }^{8}$ This imaging is also well tolerated ${ }^{9}$ since, unlike traditional dilated fundus examinations, it is less time consuming and avoids pupillary dilation, ${ }^{10}$ and vision recovers rapidly after the nonmydriatic imaging. ${ }^{11}$ Thus, in this study, subjects could view their own images immediately and see the pathological changes occurring in their own eyes. Combined with similar images of the severe complications that retinopathy can cause, this

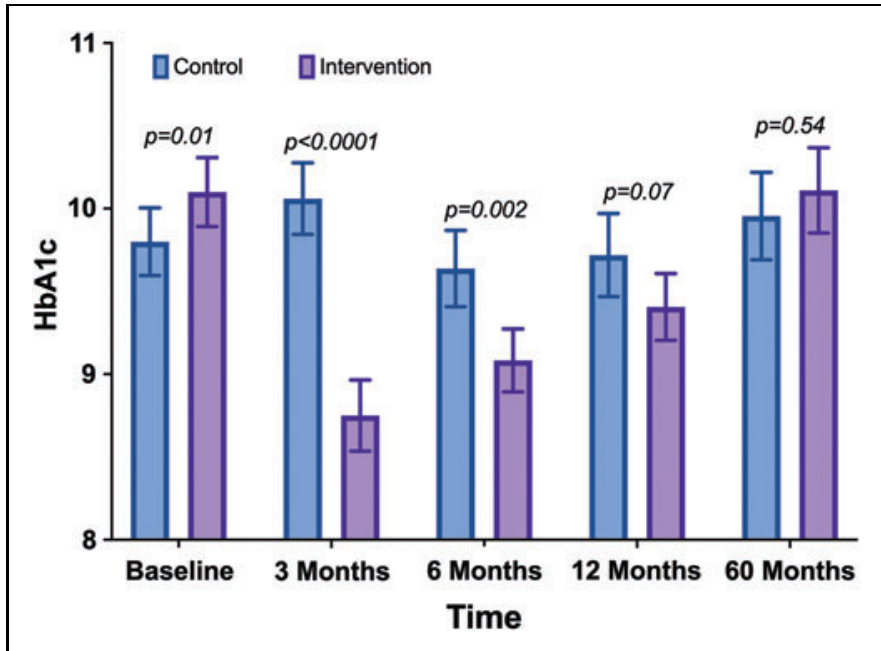

Fig. 3. Follow-up flow diagram. Color images are available online. approach may have helped personalize both the experience and the risks, thus possibly providing additional impetus for subsequent efforts to improve glycemic control.

This cohort is also unique compared to other interventional studies in that it is derived solely from an endocrinology clinic in Beirut. It is thus unlikely that the patients had seen prior images of their retina, and thus, the impact of seeing their own pathology for the first time might be particularly powerful. Furthermore, the cohort's baseline mean HbA1c was 10.0, a high value suggesting substantive risk of retinopathy progression and eventual vision loss if not appropriately addressed. This may have further increased impact of the intervention. In addition, vision loss in less developed countries often has an even greater personal and family burden than in developed societies given a relative lack of supportive services and expendable income. Finally, the physician subject interaction may be particularly strong in this cohort as evidenced by the excellent long-term follow-up.

The intervention benefits consistently diminished over time becoming statistically insignificant at some point between 6 and 12 months. These data suggest that further reinforcement with subsequent similar interventions may be needed at yearly intervals. However, it remains unknown whether repeating the same intervention would provide similar motivation and benefit over time. In addition, it should be noted that this imaging approach does not replace a comprehensive retinal ophthalmologic examination that is generally recommended at least annually for patients with diabetes when such resources are available. Whether continued encouragement to maintain recommended ophthalmological follow-up or the receipt of such ophthalmic care would modify the observed $\mathrm{HbA1c}$ response is also unknown. Changes in DR severity were not evaluated in this study due to the inadequate subject number at any given DR severity level. Furthermore, this study did not evaluate specific behaviors that may have been modified by this intervention, nor whether these behaviors could be encouraged or prolonged.

A previous study by the Diabetic Retinopathy Clinical Research Network (DRCR.net Protocol M) evaluated a scripted educational intervention at 4-month and yearly intervals. ${ }^{12}$ The intervention group did not have any $\mathrm{HbA} 1 \mathrm{c}$ 


\begin{tabular}{|c|c|c|c|c|c|c|}
\hline & $\begin{array}{l}\text { CURRENT STUDY } \\
\text { INTERVENTION }\end{array}$ & $\begin{array}{c}\text { CURRENT STUDY } \\
\text { CONTROL }\end{array}$ & $\begin{array}{l}\text { PROTOCOL M } \\
\text { FREQUENT } \\
\text { INTERVENTION }\end{array}$ & $\begin{array}{l}\text { PROTOCOL M } \\
\text { ANNUAL } \\
\text { INTERVENTION }\end{array}$ & $\begin{array}{c}\text { PROTOCOL M } \\
\text { FREQUENT } \\
\text { CONTROL }\end{array}$ & $\begin{array}{c}\text { PROTOCOL M } \\
\text { ANNUAL } \\
\text { CONTROL }\end{array}$ \\
\hline Age (years), mean & 55 & 56 & 65 & 63 & 64 & 61 \\
\hline $\begin{array}{l}\text { Diabetes duration } \\
\text { (years), median }\end{array}$ & 9 & 10 & 17 & 14 & 18 & 13 \\
\hline Hypertension (\%) & 56 & 60 & 63 & 58 & 63 & 57 \\
\hline
\end{tabular}

All $p$-values $>0.05$.

benefit. In DRCR.net Protocol M, the intervention occurred at the ophthalmologist office and involved providing the subject with their HbA1c, DR severity, brief educational discussion, and a brief knowledge assessment. Protocol M did not use the subjects own images, was performed entirely within the United States, and was not focused on underserved localities. Furthermore, as shown in Table 2, there were substantial differences in cohort characteristic between Protocol M and the current study.

Compared to Protocol M, the current study has younger subjects, higher percentage of women, shorter duration of diabetes, less hypertension, less insulin use, and higher HbA1c. Thus, it appears that the DRCR subjects may have required less intense intervention and were more aggressively treated at baseline, with less room for HbA1c improvement. Furthermore, Protocol M intervention was performed in an ophthalmology office setting, suggesting that those subjects may have had greater prior education and awareness of retinopathy. Protocol M also did not include direct review of the subjects' own retinal images.

In conclusion, for this particular cohort of diabetic patients, a single, focused, personalized retinopathy education and retinal imaging session during an endocrinology visit resulted in sustained improvement of $\mathrm{HbA1c}$ for up to 6 months. However, the benefit declined with time and was no longer evident after 5 years. Nevertheless, given that substantial reductions in ocular and other medical complications are closely associated with improvement of $\mathrm{HbA1c}$, further exploration is warranted to determine why this approach proved beneficial over this period and how to successfully extrapolate such interventions to other cohorts.

\section{Disclosure Statement}

No competing financial interests exist.

\section{Funding Information}

This project was supported, in part, from the Merck Company through a grant for research in diabetes.

\section{REFERENCES}

1. Dong $Y$, Wang $P$, Dai $Z$, Liu $K$, Jin $Y$, Li A, et al. Increased self-care activities and glycemic control rate in relation to health education via Wechat among diabetes patients: A randomized clinical trial. Medicine (Baltimore) 2018;97: e13632.

2. Bluml BM, Kolb LE, Lipman R. Evaluating the impact of year-long, augmented diabetes self-management support. Popul Health Manag 2019;22:522-528.

3. Salti H, Cavallerano JD, Salti N, Jawhari DJ, Haddad S, Sun JK, et al. Nonmydriatic retinal image review at time of endocrinology visit results in short-term $\mathrm{HbA} 1 \mathrm{c}$ reduction in poorly controlled patients with diabetic retinopathy. Telemed J E Health 2011;17:415-419.

4. Diabetes Control and Complications Trial Research Group, Nathan DM, Genuth $S$, Lachin J, Cleary P, Crofford 0 , et al. The effect of intensive treatment of diabetes on the development and progression of long-term complications in insulin-dependent diabetes mellitus. N Engl J Med 1993;329:977-986.

5. Intensive blood-glucose control with sulphonylureas or insulin compared with conventional treatment and risk of complications in patients with type 2 diabetes (UKPDS 33). UK Prospective Diabetes Study (UKPDS) Group. Lancet $1998 ; 352: 837-853$

6. Coyne KS, Margolis MK, Kennedy-Martin T, Baker TM, Klein R, Paul MD, et al. The impact of diabetic retinopathy: Perspectives from patient focus groups. Fam Pract 2004;21:447-453. 


\section{AIELLO ET AL.}

7. Cox DJ, Kiernan BD, Schroeder DB, Cowley M. Psychosocial sequelae of visual loss in diabetes. Diabetes Educ 1998;24:481-484.

8. Bursell $S E$, Cavallerano JD, Cavallerano AA, Clermont AC, Birkmire-Peters D, Aiello LP, et al. Stereo nonmydriatic digital-video color retinal imaging compared with Early Treatment Diabetic Retinopathy Study seven standard field 35-mm stereo color photos for determining level of diabetic retinopathy. Ophthalmology 2001;108:572-585.

9. Cavallerano JD, Aiello LP, Cavallerano AA, Katalinic P, Hock K, Kirby $R$, et al. Nonmydriatic digital imaging alternative for annual retinal examination in persons with previously documented no or mild diabetic retinopathy. Am J Ophthalmol 2005;140:667-673.

10. Bruce BB, Lamirel C, Biousse V, Ward A, Heilpern KL, Newman NJ, et al. Feasibility of nonmydriatic ocular fundus photography in the emergency department: Phase I of the FOTO-ED study. Acad Emerg Med 2011;18: 928-933.

11. Goel $S$, Maharajan $P$, Chua $C$, Dong B, Butcher M, Bagga P. Driving ability after pupillary dilatation. Eye (Lond) 2003;17:735-738.

12. Aiello LP, Ayala AR, Antoszyk AN, Arnold-Bush B, Baker C, Bressler NM, et al. Assessing the effect of personalized diabetes risk assessments during ophthalmologic visits on glycemic control: A randomized clinical trial. JAMA Ophthalmol 2015;133:888-896.

Address correspondence to:

Haytham I. Salti, MD

Department of Ophthalmology

American University of Beirut Medical Center

Cairo Street, Hamra, P.O. Box 11-0236, Riad El Solh

Beirut 11072020

Lebanon

E-mail: hs06@aub.edu.lb

Received: September 11, 2019

Accepted: October 23, 2019

Online Publication Date: January 14, 2020 